\title{
Malignant melanoma with a rhabdoid phenotype exhibiting numerous solid tumor masses: A case report
}

\author{
TOMOKI NAKAMURA ${ }^{1}$, AKIHIKO MATSUMINE ${ }^{1}$, HIROAKI KATO ${ }^{1}$, KATSUYUKI KUSUZAKI $^{2}$, \\ KEISUKE NISHIMURA ${ }^{3}$, TETSUYA MURATA ${ }^{4}$, TAIZO SHIRAISHI ${ }^{3}$, YOSHINAO ODA ${ }^{5}$, \\ MASAZUMI TSUNEYOSHI $^{5}$ and ATSUMASA UCHIDA ${ }^{1}$ \\ ${ }^{1}$ Department of Orthopaedic Surgery, Mie University Postgraduate School of Medicine; ${ }^{2}$ Department of \\ Orthopaedic Surgery, JA Odai Kosei Hospital; ${ }^{3}$ Department of Pathology, Mie University Postgraduate School \\ of Medicine; ${ }^{4}$ Department of Pathology and Laboratory Medicine, JA Suzuka General Hospital; \\ ${ }^{5}$ Department of Pathology, Kyushu University Postgraduate School of Medicine, Japan
}

Received October 7, 2008; Accepted January 7, 2009

DOI: 10.3892/or_00000299

\begin{abstract}
We present a case of malignant melanoma with a rhabdoid phenotype in a 44-year-old female with a quite unique and aggressive clinical course. Rhabdoid features are defined by characteristics such as sheets or solid trabeculae of neoplastic cells with large, vesicular, round to bean-shaped nuclei, prominent centrally located nucleoli, and abundant eccentric cytoplasm. Although various histological differential diagnoses were cited for the present case that showed 'rhabdoid features', most of them were excluded on the basis of the clinical history, tumor location, clinical behavior, and a broad panel of immunohistochemical stains. In the present case, the immunohistochemical findings were positive for vimentin, S-100 protein, melan-A, and EMA, but negative for HMB45, cytokeratin, CD34 and desmin. In addition, the positive expression of BAF47 was also recognized. These findings lead to the conclusion that this quite unique aggressive soft tissue tumor should therefore be diagnosed as malignant melanoma with a rhabdoid phenotype.
\end{abstract}

\section{Introduction}

Malignant melanoma is known to display tremendous histologic diversity $(1,2)$. Rare cases of malignant melanoma with a rhabdoid phenotype have been reported (3-7). We present a case of malignant melanoma with a rhabdoid phenotype which has a quite unique and aggressive clinical course.

Correspondence to: Dr Akihiko Matsumine, Department of Orthopaedic Surgery, Mie University Postgraduate School of Medicine, Edobashi2-174, Tsu-city, Mie 514-8507, Japan

E-mail: matsumin@clin.medic.mie-u.ac.jp

Key words: soft tissue sarcoma, malignant melanoma, rhabdoid phenotype, rhabdoid cell

\section{Patients and methods}

Clinical history. A 44-year-old female attended the hospital because of multiple subcutaneous tumor masses which were initially noticed 2 months earlier and were continuously increasing in number and size. Numerous masses were distributed throughout the body (Fig. 1). The tumor masses were elastic hard, well-circumscribed with smooth surfaces, and fixed to the deep fascia. The patient's laboratory examination revealed decreased hemoglobin, total protein and albumin $(9.2,6.3$ and $3.1 \mathrm{~g} / \mathrm{dl}$, respectively) and increased $\mathrm{LDH}, \mathrm{C}$-reactive protein and soluble-IL2 receptor (1179 IU/1, $3.39 \mathrm{mg} / \mathrm{dl}$ and $864.2 \mathrm{U} / \mathrm{ml}$, respectively). Other tumor makers including CEA, AFP and CA125 were within the normal range. Fluorodeoxyglucose-positron emission tomography (FDG-PET) revealed numerous positive lesions throughout the body (Fig. 2). Most of the tumors were in the subcutaneous tissue partially involving the deep fascia of the muscle. Magnetic resonance imaging (MRI) indicated that the tumors located at right anterior shoulder, left sternoclavicular joint and left axilla, showed an iso-signal intensity on T1-weighted images and heterogeneously high-signal intensity on T2-weighted images. After gadolinium contrast medium administration, the tumors were heterogeneously enhanced (Fig. 2). An excision biopsy was performed on the lesions of the right anterior shoulder and abdominal wall, both of which were causing severe pain.

Tissue was fixed in $10 \%$ neutral-buffered formalin, embedded in paraffin, and stained with hematoxylin and eosin. For the immunohistochemical analysis, the primary antibodies listed in Table I were used.

\section{Results}

The tumor masses had a yellowish, homogeneous cut surface with small areas of hemorrhage and necrosis. Microscopic findings showed a proliferation of round or polygonal cells with vesicular nuclei, prominent nucleoli and eosinophilic cytoplasm, arranged in sheets or alveolar patterns, accompanied by necrosis. Rhabodid cells with cytoplasmic inclusion 
Table I. Immunohistochemical stain.

\begin{tabular}{lrlr}
\hline Antibody & Dilution & source & Pretreatment \\
\hline S-100 protein & $1: 100$ & Dako Corporation, Tokyo, Japan & 10 min Microwave \\
Vimentin & $1: 100$ & Dako & 10 min Microwave \\
Melan-A (A103) & $1: 25$ & Dako & 20 min Microwave \\
BAF47 (25) & $1: 250$ & BD Transduction Laboratories, San Diego, CA, USA & 10 min Microwave \\
EMA (E29) & $1: 100$ & Dako & 10 min Microwave \\
HMB-45 & $1: 75$ & Dako & 10 min Microwave \\
Pan-CK (5D3) & $1: 100$ & Novocastra Laboratories, Newcastle, UK & 10 min Microwave \\
CK (CAM5.2) & $1: 100$ & Dako & 10 min Microwave \\
LCA (CD45) & $1: 50$ & Dako & 10 min Microwave \\
Smooth muscle actin (IA4) & $1: 400$ & Dako & 10 min Microwave \\
$\kappa$ light chain & $1: 50$ & Dako & 10 min Microwave \\
$\lambda$ light chain & $1: 50$ & Dako & 10 min Microwave \\
CD34 (QBEnd 10) & $1: 40$ & Dako & 10 min Microwave \\
Desmin (D33) & $1: 200$ & Dako &
\end{tabular}

EMA, epithelial membrane antigen; Pan-CK, pan-cytokeratine; CK, cytokeratine; LCA, leukocyte common antigen.

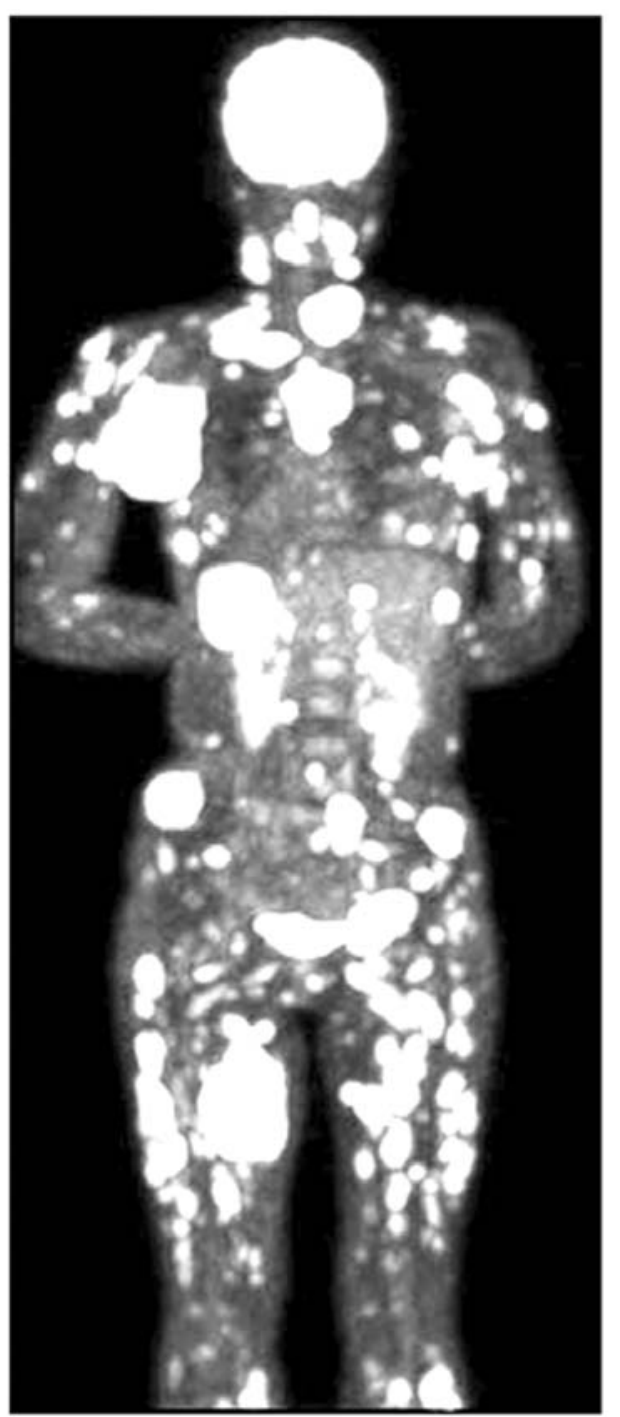

Figure 1. PET revealed numerous tumors throughout the body.
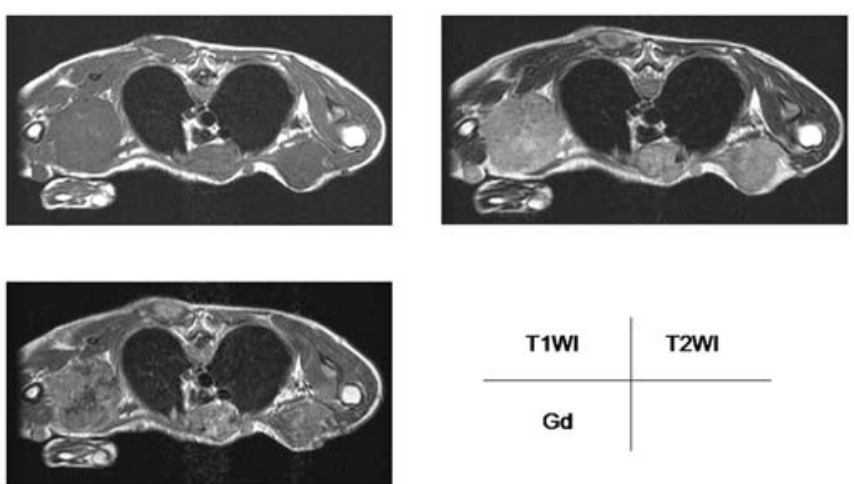

Figure 2. MRI of the bilateral axillary (left: T1WI, middle: T2WI and right: Gd-enhance).

bodies were prominent through the tumor (Fig. 3). Melanosome were absent through the tumor. Since malignant tumors with rhabdoid feature were suspected, a further immunohistochemical analysis was performed. The immunohistochemical analysis showed the tumors to be diffusely positive for vimentin and S-100 protein; focally positive for Melan-A and epithelial membrane antigen (EMA); negative for HMB45, pan-cytokeratin, CAM5.2, CD34, $\lambda$ light chain, $\kappa$ light chain and desmin. In addition, the positive expression of BAF47 (SMARCB1/INI1) was also recognized (Fig. 4). These unusual aggressive tumors were diagnosed as malignant melanoma with a rhabdoid phenotype. Although we worked up the detection of primary lesion, especially of the eye and internal organs, we failed to demonstrate a primary melanoma. The patient underwent systemic chemotherapy using dacarbazin: $230 \mathrm{mg} / \mathrm{m}^{2}$, i.v. $(1 \mathrm{~h})$, day 1-3 (Dacarbazin ${ }^{\circledR}$ : Kyouwahakkou Co, Ltd Tokyo, Japan), nimustine hydrochloride: $60 \mathrm{mg} / \mathrm{m}^{2}$, day 1 (Nidran ${ }^{\circledR}$ : Daiichi-Sankyo Co. Ltd Tokyo, Japan), cisplatin $25 \mathrm{mg} / \mathrm{m}^{2}$, i.v. (4 h) day 1-3 

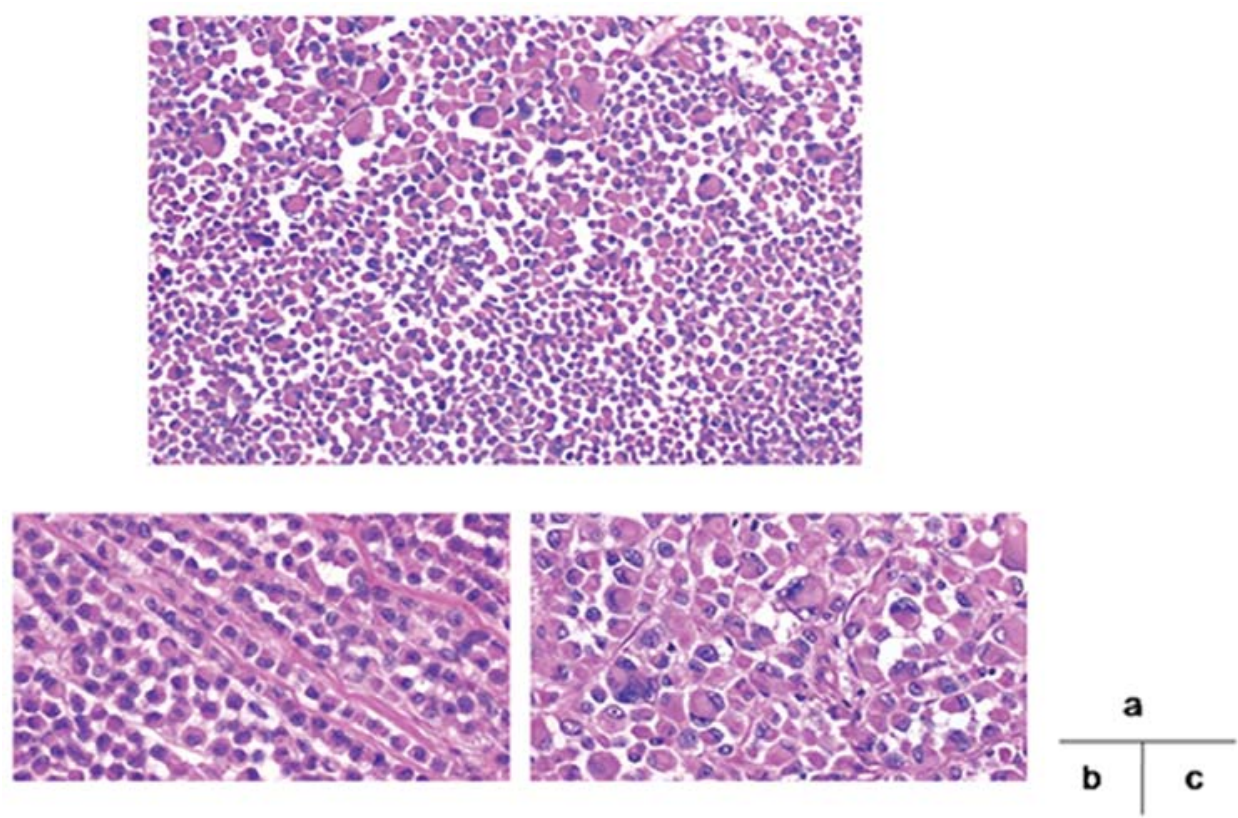

Figure 3. H\&E stain (upper: x20, lower: x40): Microscopically, tumor is composed of a diffuse proliferation of rounded or polygonal cells arranged in sheets (a) and alveolar (b) pattern. Tumor cells disclose characteristic glassy eosinophilic cytoplasm, eccentric vesicular nuclei and prominent nucleoi (c).

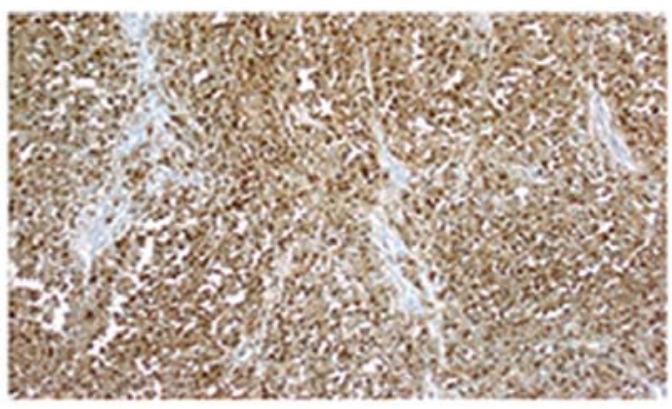

a

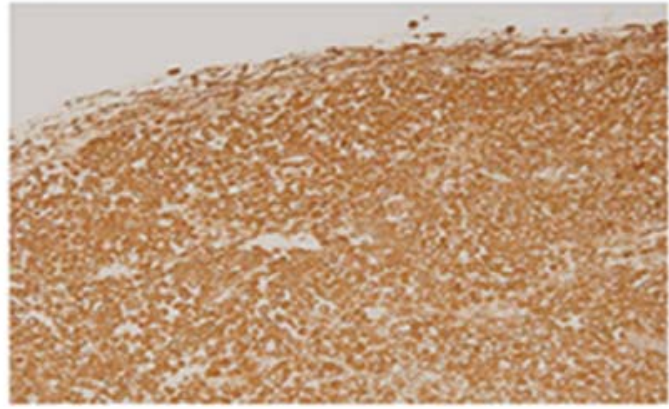

C

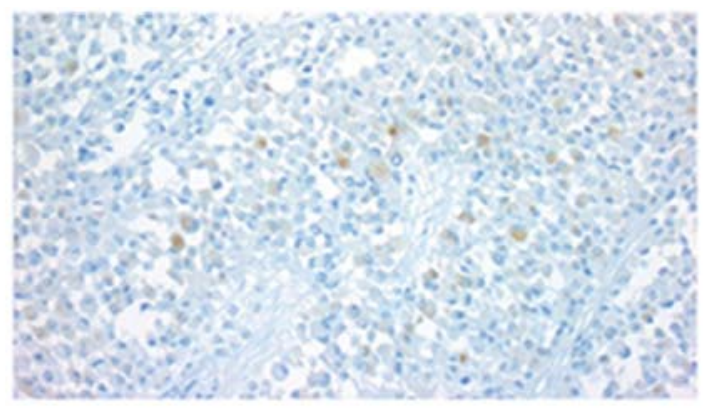

b

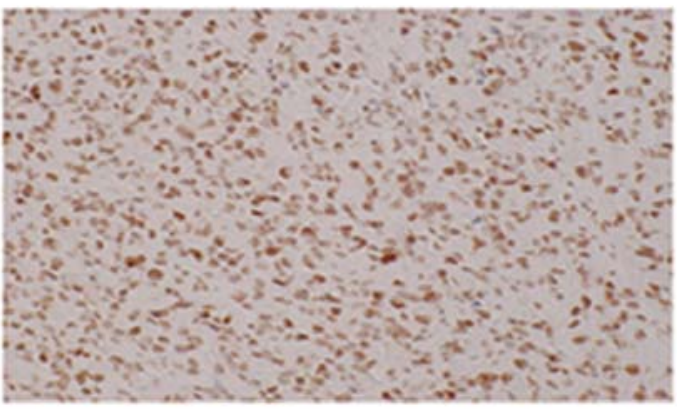

d

Figure 4. Immunohistochemical staining: (a) strong S100 protein expression by the neoplastic cells, (b) focally positive staining for Melan-A, (c) diffuse positive staining for vimentin and (d) diffuse BAF47 expression by the neoplastic cells.

(Randa ${ }^{\circledR}$ : Nippon Kayaku Co. Ltd, Tokyo, Japan), but no reduction of the tumors was observed. Since the tumor mass of the left axillary region increased in size, and caused severe neuralgia, radiation therapy was performed at a dose of 36 Gy/9 fractions.

Two months after the excision biopsy, the patient died of an intracranial hemorrhage from a metastatic brain tumor. Permission to perform an autopsy was rejected by the family.

\section{Discussion}

The microscopic findings of the present case showed a proliferation of rounded or polygonal cells with vesicular nuclei, prominent nucleoli, and eosinophilic cytoplasm, arranged in sheets or alveolar patterns, accompanied with necrosis. Rhabdoid cells with cytoplasmic inclusion bodies were prominent throughout the tumor. The term 'rhabdoid' is 
generally used when the microscopic findings resemble rhabdomyoblasts, although muscle markers are absent. Rhabdoid features are defined by characteristics such as morphological findings, sheets or solid trabeculae of neoplastic cells with large, vesicular, round to bean-shaped nuclei, prominent centrally located nucleoli, and abundant eccentric cytoplasm. Mitotic figures were frequently seen, and tumor necrosis was common. Initially, malignant rhabdoid tumors (MRT) were categorized among pediatric renal neoplasms as a rhabdomyosarcomatous variant of Wilms tumor in a pediatric renal neoplasm, but it was later re-categorized into a malignant rhabdoid tumor (MRT) as a separate entity $(8,9)$. Thereafter, numerous cases of MRT arising from extra-renal sites including the liver $(10,11)$, brain $(10,11)$, skin (11) and soft tissue (10-13) have been reported. Subsequent studies revealed the rhabdoid cells have also been seen in other tumors, such as some carcinomas $(14,15)$, sarcomas (16-18), in the central nervous system (19) and malignant melanoma $(6,7)$. The rhabdoid features are thought to represent a common dedifferentiated end point for a variety of neoplasms. Therefore 'rhabdoid features' may be a term which represents phenotypes of malignant neoplasms (20-22). The histological findings of the present case showed the characteristic features of rhabdoid cells.

Although various histological differential diagnoses were cited for the present case that showed 'rhabdoid features', most of them were excluded on the basis of the clinical history, tumor location, clinical behavior, and a broad panel of immunohistochemical stains. First, epithelioid sarcoma, which usually affects the distal extremities, may have rhabdoid cells. However, in the present case, the number of rhabdoid cells was small and they were focally recognized. Epithelioid sarcoma is commonly diffusely positive for the CK (17). Additionally, it is not usually as aggressive as the present case. Second, rhabdomyosarcoma with extensive rhabdomyoblastic differentiation may look morphologically similar to the present case, but usually shows diffusely positive staining for myogenic makers (18).

Finally, extra-renal-MRT (EMRT), which has overlapping features with malignant melanoma with a rhabdoid phenotype $(6,7)$ could be cited. Certainly, the immunohistochemical characteristics overlapped with EMRT in the present case including the diffusely positive S-100 protein and vimentin marker, and the negative staining for desmin, CD34, cytokeratin and LCA marker. Recently, subsequent studies have consistently found deletions or mutations of the INI1 gene (also known as hSNF5/SMARCB/BAF47) in the vast majority of MRT and EMRT, although overall up to $20 \%$ of rhabdoid tumors of all sites show no evidence of INI1 gene alterations $(23,24)$. Therefore, a negative immunohistochemical reaction for a monoclonal anti-SMARCB1/ hSNF/INI1 antibody (BAF47) has been proposed to diagnose rhabdoid tumors $(6,11,14)$. The present case, however, showed positive BAF47 staining. In addition, the majority of the cases of EMRT were infants or children (12).

In the present case, the immunohistochemical findings were positive for vimentin, S-100 protein, melan-A, and EMA, but negative for HMB45, cytokeratin (CAM5.2, AE1/AE3), CD34 and desmin. In addition, the positive expression of BAF47 (SMARCB1/INI1) was also recognized. These immunoprofiles were similar to that of previous studies of malignant melanoma with a rhabdoid phenotype (3-7), namely, positive for S-100 protein, vimentin and EMA. However, Laskin et al reported that malignant melanoma with a rhabdoid phenotype was negative for S-100 protein, unlike with the present case (5). One explanation is that this discrepancy might be attributed the differences of the specificities of the antibodies used. Furthermore, this variability in immunohistochemical features is frequent among the rhabdoid features which represent a common dedifferentiated end point for a variety of neoplasms (3-7,15-17). Further molecular and cytogenetic studies of the differentiation and the dediffer-entiation of the neoplasm are warranted.

All these findings lead to the conclusion that this quite unique aggressive soft tissue tumor should be diagnosed as a malignant melanoma with a rhabdoid phenotype.

\section{References}

1. Banerjee SS and Harris M: Morphological and immunophenotypic variations in malignant melanoma. Histopathology 36: 387-402, 2000.

2. Pizzichetta MA, Talamini R, Stanganelli I, et al: Amelanotic/ hypomelanotic melanoma: clinical and dermoscopic features. $\mathrm{Br}$ J Dermatol 150: 1117-1124, 2004

3. Bittesini L, Dei Tos AP and Fletcher CDM: Metastatic malignant melanoma showing a rhabdoid phenotype: further evidence of a non-specific histological pattern. Histopathology 20: 167-170, 1992.

4. Borek BT, McKee PH, Freeman JA, Maguire B, Brander WL and Calonje E: Primary malignant melanoma with rhabdoid features: a histologic and immunocytochemical study of three cases. Am J Dermatopathol 20: 123-127, 1998.

5. Laskin WB, Knittel DR and Frame JN: S-100 protein and HMB45 negative 'rhabdoid' malignant melanoma: a totally dedifferentiated malignant melanoma? Am J Clin Pathol 103: 772-773, 1995.

6. Chang ES, Wick MR, Swanson PE and Dehner LP: Metastatic malignant melanoma with rhabdoid features. Am J Clin Pathol 102: 426-431, 1994.

7. Abbott JJ, Amirkman RH and Hoang MP: Malignant melanoma with a rhabdoid phenotype. Arch Pathol Lab Med 128: 686-688, 2004.

8. Beckwith JB and Palmar NF: Histopathology and prognosis of Wilm's tumor: Results from the First National Wilm's Tumor Study. Cancer 41: 1937-1948, 1978.

9. Haas JE, Palmer NF, Weinberg AG and Beckwith JB: Ultrastructure of malignant rhabdoid tumor of the kidney: A distinctive renal tumor of children. Hum Pathol 12: 646-657, 1981

10. Sotelo-Avila C, Gonzalez-Crussi F, deMello D, et al: Renal and extrarenal rhabdoid tumors in children: A clinocopathologic study of 14 patients. Semin Diagn Pathol 3: 151-163, 1986.

11. Parham DM, Weeks DA and Beckwith JB: The clinicopathologic spectrum of putative extrarenal rhabdoid tumors. An analysis of 42 cases studied with immunohistochemistry or electron microscopy. Am J Surg Pathol 18: 1010-1029, 1994.

12. Oda Y and Tsuneyoshi M: Extrarenal rhabdoid tumors of soft tissue: Clinicopathological and molecular genetic review and distinction from other soft-tissue sarcomas with rhabdoid features. Pathol Int 56: 287-295, 2006.

13. Franburg-Smith JC, Hengge M, Hengge UR, Smith JS Jr and Miettinen M: Extrarenal rhabdoid tumors of soft tissue: A clinicopathologic and immunohistochemical study of 18 cases. Ann Diagn Pathol 2: 351-362, 1998.

14. Ueyama T, Nagai E, Yao T and Tsuneyoshi M: Vimentin-positive gastric carcinomas with rhabdoid features. A clinicopathologic and immunohistochemical study. Am J Surg Pathol 17: 813-819, 1993.

15. Cavazza A, Colby RV, Tsokos M, Rush W and Travis WD: Lung tumors with a rhabdoid phenotype. Am J Clin Pathol 105: 182-188, 1996.

16. Oshiro Y, Shiratsuchi H, Tamiya S, Oda Y, Toyoshima S and Tsuneyoshi M: Rhabdoid features in leiomyosarcoma of soft tissue: With special reference to aggressive behavior. Med Pathol 13: $1211-1218,2000$. 
17. Miettinen M, Fanburg-Smith JC, Virolainen M, Shmookler BM and Fetsch JF: Epithelioid sarcoma: An immunohistochemical analysis of 112 classical and variant cases and a discussion of the differential diagnosis. Hum Pathol 30: 934-942, 1999.

18. Oda Y, Miyajima K, Kawaguchi K, et al: Pleomorphic leiomyosarcoma. Clinicopathologic and immunohistochemical study with special emphasis on its distinction from ordinary leiomyosarcoma and malignant fibrous histiocytoma. Am J Surg Pathol 25: 1030-1038, 2001.

19. Perry A, Scheithauer BW, Stafford SL, Abell-Aleff PC and Meyer FB: 'Rhabdoid' meningioma. An aggressive variant. Am J Surg Pathol 22: 1482-1490, 1998.

20. Ogino S, Ro JY and Redline W: Malignant rhabdoid tumor: A phenotype? An entity? - A controversy revisited. Adv Anat Pathol 7: 181-190, 2000.
21. Weeks DA, Beckwith JB and Mierau GW: Rhabdoid tumor: an entity or a phenotype? Arch Pathol Lab Med 113: 113-114, 1989.

22. Wick MR, Ritter JH and Dehner LP: Malignant rhabdoid tumors: A clinicopathologic review and conceptual discussion. Semin Diagn Pathol 12: 233-248, 1995.

23. Bourdeaut F, Fréneaux P, Thuille B, et al: hSNF5/INI1deficient tumors and rhabdoid tumours are convergent but not fully overlapping entities. J Pathol 211: 323-330, 2007.

24. Hoot AC, Russo P, Judkins AR, Perlman EJ and Biegel JA: Immunohistochemical analysis of hSNF5/INI1 distinguishes renal and extra-renal malignant rhabdoid tumors from other pediatric soft tissue tumors. Am J Surg Pathol 28: 1485-1491, 2004. 